\title{
Self-Organization on Multiple Length Scales in "Hairy Rod"-Coil Block Copolymer Supramolecular Complexes
}

\author{
Matthew R. Hammond, Harm-Anton Klok, Raffaele Mezzenga*
}

A peptide-synthetic hybrid block copolymer, poly(ethylene oxide)-block-poly(t-glutamic acid), is shown to form supramolecular complexes with primary alkylamines of varying alkyl chain lengths (8 to 18 methylene units) in organic solvents via acid-base proton transfer and subsequent ionic bonding. The peptidic block being in the $\alpha$-helical conformation, these materials behave as coil"hairy rod" block copolymers, and show hierarchically selforganized nanostructures in the solid state. X-ray scattering measurements show mesomorphic behavior at the length scales of both the overall block copolymer and the polypeptide-alkylammonium complex.

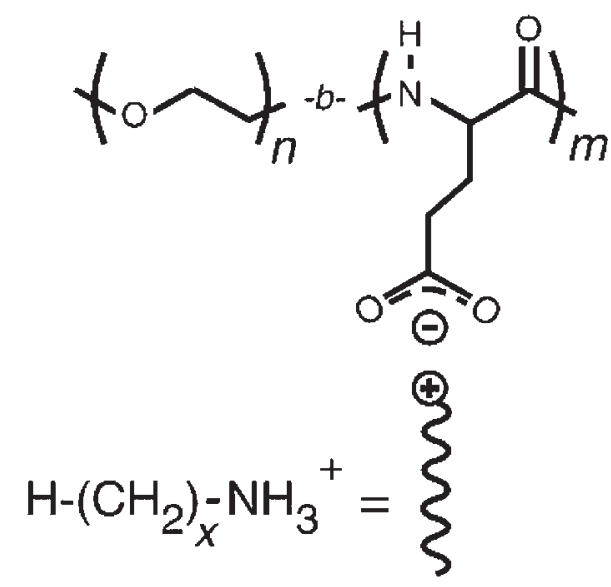

\section{Introduction}

The last two decades have seen an explosion of research activity in the area of self-assembled polymeric and supramolecular materials. ${ }^{[1-3]}$ Self-assembly schemes rely on an often delicate balance between competing repulsive and attractive forces between structural elements. In traditional coil-coil block copolymers, microphase separated structures are dictated by the balance of immisci-

M. R. Hammond, R. Mezzenga

Department of Physics and Fribourg Center for Nanomaterials, University of Fribourg, Chemin du Musée 3, 1700 Fribourg, Switzerland

Fax: +41 (o)26300 9747; E-mail: raffaele.mezzenga@unifr.ch H.-A. Klok

École Polytechnique Federale de Lausanne, Institut des Matériaux, Laboratoire des Polymères, Batiment MXD, Station 12, $\mathrm{CH}-1015$ Switzerland

R. Mezzenga

Nestlé Research Center, Vers-chez-les-blanc, 1000 Lausanne 26, Switzerland bility between blocks and the stretching of chains confined at the microphase interface due to covalent linkages. ${ }^{[4]}$ Mesomorphic behavior is also observed in polymeric comb-like supramolecules created by bonding mesogenic or flexible side chains to a polymer backbone through non-covalent physical interactions such as ionic bonding, ${ }^{[5-10]}$ hydrogen bonding, ${ }^{[11,12]}$ metal coordination ${ }^{[13]}$ or various combinations thereof. ${ }^{[14,15]}$

Increasingly complicated schemes for constructing self-organizing systems now incorporate multiple building blocks with various structural motifs, taking advantage of competing interactions acting on multiple length scales, and thus giving rise to hierarchical self-assembly. ${ }^{[3]}$ In block copolymers where one block is rod-like, whether owing to helicity or extended $\pi$-conjugation, the propensity of the rods to order in liquid crystalline arrangements offers an additional ordering principle. ${ }^{[16-20]}$ In this case, order can be observed on the scale of a few $\mathrm{nm}$ (inter-rod liquid crystalline packing) and on the larger length scale of the block copolymer, typically a few tens of nm. Rod-coil block copolymers where the rod block is an $\alpha$-helical 
polypeptide are particularly interesting, not only as materials bridging the interface between synthetic polymers and biological systems, ${ }^{[21]}$ but also due to the potential for stimuli-responsive changes in the polypeptide secondary structure.

Hierarchical ordering has also been demonstrated by combining coil-coil block copolymers with the supramolecularly complexed polymer-surfactant systems described above. ${ }^{[10,15]}$ The incorporation of polypeptidebased block copolymers into these schemes has only recently begun to be explored. Hadjichristidis, Ikkala et al. showed hierarchical ordering in complexes between sulfonic acid based surfactants and the poly(L-lysine) block of a poly( $\gamma$-benzyl-L-glutamate)-block-poly(L-lysine) (PBLGPLL) block copolymer. ${ }^{[22]}$ Structural hierarchy observed in these samples involved lamellar ordering on the block copolymer length scale, packing of PBLG $\alpha$-helical rods and either hexagonal or lamellar arrangement within the PLL(surfactant) complex domains, depending on surfactant type. Thus the materials were appropriately described as "rod-comb" block copolypeptide-surfactant complexes.

In this communication, we report on the solid state structures observed in polymeric complexes formed from a poly(ethylene oxide)-poly(L-glutamic acid) (PEO-PLGA) block copolymer and primary $n$-alkyl amines with alkyl tail lengths varying from 8 to 18 carbons. Analogous complexation schemes have previously been reported (e.g., complexation of alkyl carboxylic acid amphiphiles to the amine groups of PEO-block-poly(ethylene imine) copolymers $\left.{ }^{[10]}\right)$. The supramolecular complexes presently considered, best described schematically as "hairy rod"-coil
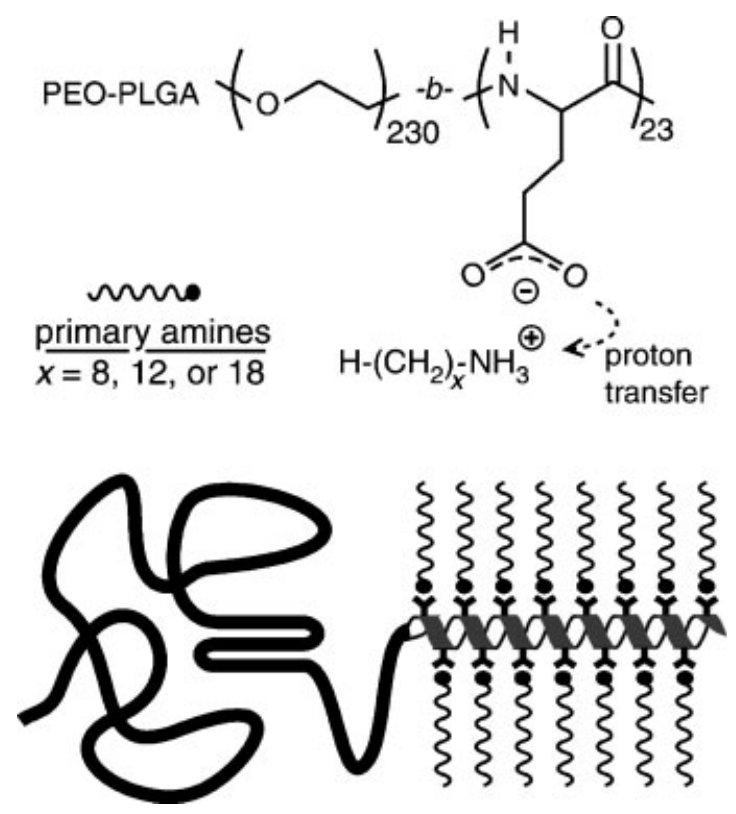

Scheme 1. Schematic drawing of the PEO-PLGA $\left(C_{x}\right)$ complexes considered in this work. structures, are represented in Scheme 1. Although studies of ionic complexes of PEO-polypeptide block copolymers and oppositely charged small molecules have been reported, ${ }^{[23,24]}$ and notwithstanding the block copolypeptide "rod-comb" structures described above (ref. ${ }^{[22]}$ ), we believe this to be the first report showing hierarchical ordering in the solid state structures of such "hairy rod"-coil peptide-synthetic block copolymer-surfactant supramolecular complexes. The various complexes are hereafter referred to using the notation PEO-PLGA $(C x)$, where $x$ represents the number of methylene units in the amine amphiphiles.

\section{Experimental Part}

\section{Synthesis of Poly(ethylene oxide)-block-Poly(I-glutamic acid)}

$\mathrm{PEO}_{230}$-PLGA 23 block copolymer was prepared by removal of benzyl protecting groups from a parent PEO-block-poly $(\gamma$-benzylL-glutamate) $\left(\mathrm{PEO}_{230}-\mathrm{PBLG}_{23}\right)$ block copolymer (the subscripts refer to the number-average degrees of polymerization for the blocks). The synthesis of the PEO-PBLG followed procedures described previously $^{[25]}$ for the ring-opening polymerization (ROP) of $\gamma$-benzyl---glutamate $\mathrm{N}$-carboxyanhydride (prepared according to ref. ${ }^{[26]}$ ), starting from amine-terminated PEO macroinitiators. In this case, $\alpha$-methoxy- $\omega$-amino-PEO (Rapp Polymere $\mathrm{GmbH}$, $\bar{M}_{\mathrm{n}}=10000 \mathrm{~g} \cdot \mathrm{mol}^{-1}$ ) was dried in vacuum prior to initiation of the ROP, but was otherwise used as received. In order to avoid any possible racemization of the peptide block, the deprotection was carried out by catalytic hydrogenation ${ }^{[27]}$ in dry DMF, with complete removal of benzyl protecting groups confirmed by ${ }^{1} \mathrm{H}$ NMR. GPC analysis of the PEO-PBLG polymer indicated that a small but not insignificant amount of PEO homopolymer (which presumably failed to initiate an ROP) remained in the block copolymer sample. Otherwise, the GPC results showed for the block copolymer a monomodal molecular weight distribution typical for this type of polymerization. To determine the number-average degree of polymerization of the block copolymer, a small sample of PEO-PLGA was purified by preparative GPC and analyzed by ${ }^{1} \mathrm{H}$ NMR (ratio of PEO protons to $\mathrm{H}_{\gamma}$-Glu and $\mathrm{H}_{\beta}$-Glu protons). Good agreement with the initial reactant stoichiometry was found.

\section{Preparation of PEO-PLGA(alkylamine) Complexes}

Complexes were prepared by mixing $\approx 1$ wt.-\% solutions of PEO-PLGA and $n$-alkylamines [octadecylamine, dodecylamine and octylamine, Fluka (Puriss), used as received] in reagent grade THF. In order to ensure complete complexation, a slightly greater than stoichiometric amount (105-110\%) of the amine was added with respect to PLGA carboxylic acid groups. The complex was then precipitated in heptane in order to remove non-complexed alkylamine, and dried in a vacuum at room temperature. Following re-dissolution of the complexes in chloroform, the final solid samples were obtained by slow solvent evaporation, followed by treatment in a vacuum at room temperature for 
$>2 \mathrm{~h}$ to ensure the removal of any remaining solvent traces. Near-full complexation (>90\% amine groups to PLGA monomers) was confirmed by ${ }^{1} \mathrm{H}$ NMR.

\section{FT-IR and Circular Dichroism Spectroscopy}

Infrared spectra were obtained with a Bruker Tensor 27 FTIR spectrometer in attenuated total reflection (ATR) mode, using a ZnSe crystal. The solid samples were pressed directly onto the crystal and spectra recorded between $600-4000 \mathrm{~cm}^{-1}$. Circular dichroism (CD) experiments were performed with a Jasco J-715 spectropolarimeter. Films for $\mathrm{CD}$ analysis were solution cast from chloroform onto quartz optical windows.

\section{Wide- and Small-Angle X-ray Scattering (WAXS and SAXS)}

WAXS and SAXS diffractograms were recorded using an AntonParr SAXSess system. The system uses a sealed tube $\mathrm{Cu} \mathrm{K} \alpha$ $(\lambda=1.542 \AA)$ source and is operated with a Kratky block camera, with a line-collimated primary beam. The scattering pattern is recorded on an image plate located $26.33 \mathrm{~cm}$ from the sample, allowing the measurement of scattering in the range $0.08 \mathrm{~nm}^{-1}<q<25 \mathrm{~nm}^{-1}$. Samples were held in place between two thin mica sheets, the background scattering from which was duly subtracted.

\section{Results and Discussion}

Complexation proceeds by acid-base proton transfer from the carboxylic acid group to the amine, followed by ionic bonding between the resulting carboxylate and alkylammonium species, as represented in Scheme 1 . The process

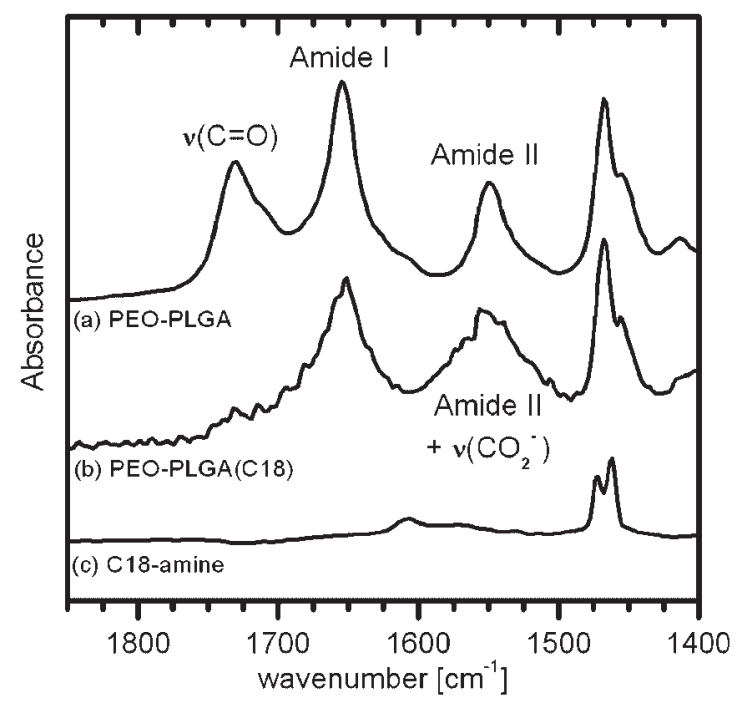

Figure 1. FT-IR spectra showing the effect of complexation of PEO-PLGA with primary alkylamines. Spectra are from solid samples of: (a) PEO-PLGA; (b) PEO-PLGA(C18); (c) octadecylamine.

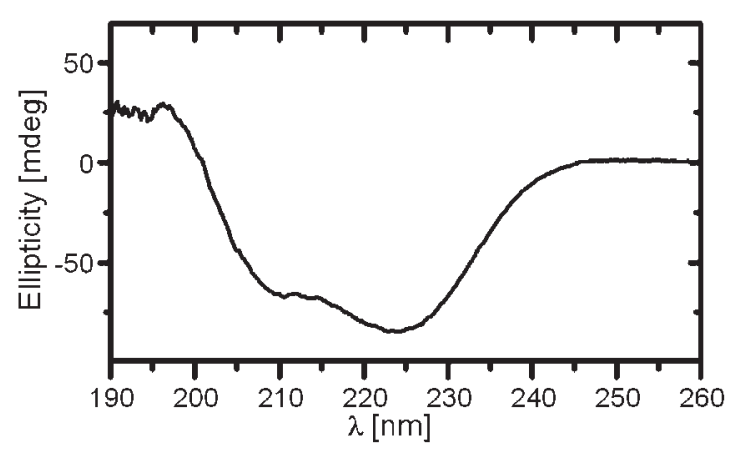

Figure 2. Circular dichroism spectrum for PEO-PLGA(C18) film cast on quartz substrate.

was monitored by FT-IR, where the carboxyl peak at $1735 \mathrm{~cm}^{-1}$ of uncomplexed PLGA [Figure 1, curve (a)] completely disappears in the case of the polymer complexed with octadecylamine, and is replaced by the asymmetric carboxylate peak in the neighborhood of $1560 \mathrm{~cm}^{-1}$ [Figure 1, curve (b)]. This latter peak overlaps with the amide II band from the polypeptide backbone; however, its presence is confirmed by comparing the relative intensity of the Amide I and II bands in the uncomplexed and complexed cases. PEO-PLGA(C12) and PEO-PLGA(C8) complexes show identical results in these respects (data not shown). The FT-IR results also give information about the secondary structure of the peptide block, as the positions of the Amide I and II bands are sensitive to the hydrogen bonding states of the peptide backbone. In all cases, before and after complexation, the Amide I and II bands appear at 1654 and $1549 \mathrm{~cm}^{-1}$, respectively, suggesting an $\alpha$-helical secondary structure. ${ }^{[28]}$ To get more concrete secondary structural information, circular dichroism spectra were recorded for films of the PEO-PLGA(CX) complexes, a representative example of which is given in Figure 2. In all cases, the signs and positions of the major bands in the spectra correspond to an $\alpha$-helical configuration of the PLGA(Cx) block. ${ }^{[28]}$

Information about the hierarchical organization within the PEO-PLGA $(C x)$ complexes, at length scales ranging from angstroms to a few tens of nanometers, was obtained from combined SAXS/WAXS measurements. Results at small angles are presented in Figure $3(\mathrm{a}-\mathrm{b})$. Uncomplexed PEO-PLGA [Figure 3(a)] shows no evidence of microphase separation. Indeed, given the propensity of carboxylic acids to hydrogen bond to $\mathrm{PEO},{ }^{[29]}$ it is reasonable to expect miscibility between the PEO and uncomplexed PLGA blocks. In contrast, the complexes [Figure 3(b)], plotted here applying the Lorentz correction in order to emphasize the peaks at the smallest angles), ${ }^{[30] a}$ show ordering on

\footnotetext{
a The Lorentz correction must not be applied to SAXS data which does not already show periodicity peaks before correction. See associated reference.
} 
(a)

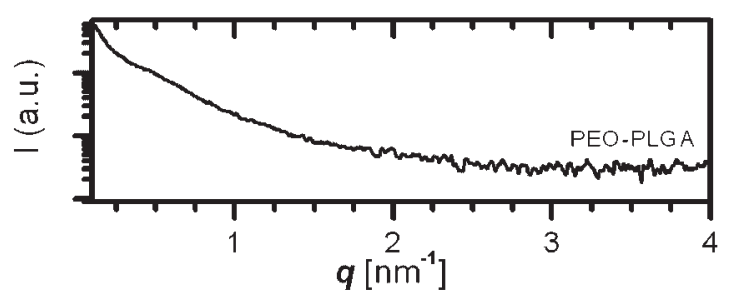

(b)

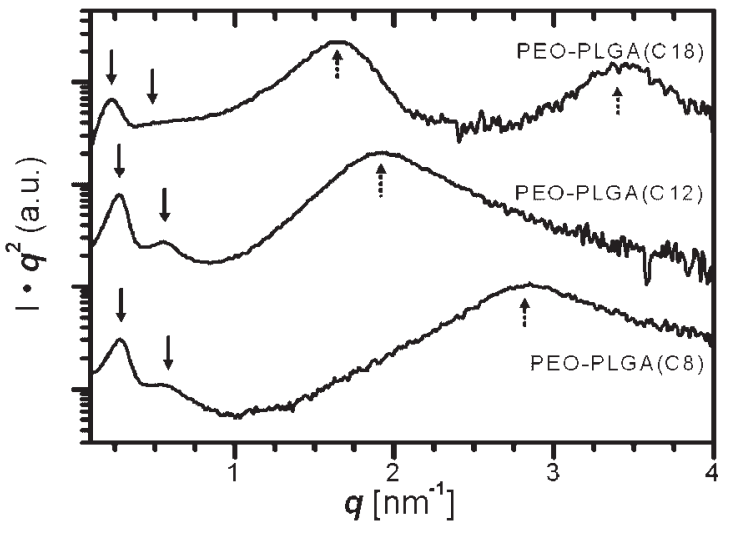

(c)

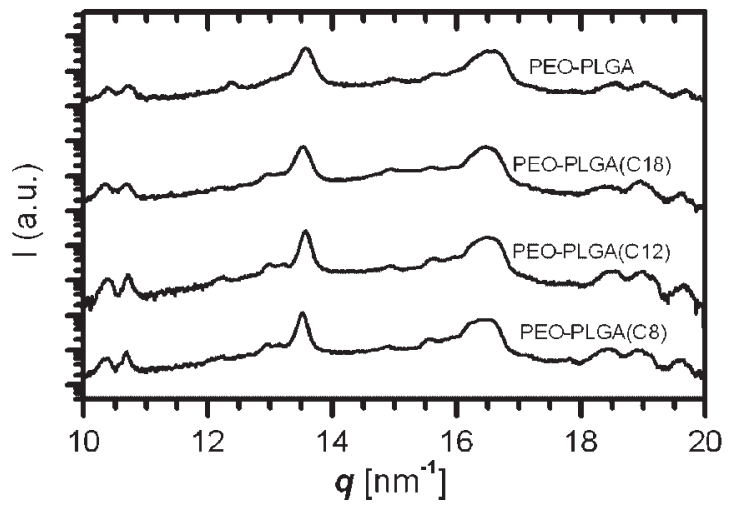

Figure 3. (a) Small-angle X-ray scattering result for uncomplexed PEO-PLGA solid (shown as intensity versus $q$ ) indicating no microphase separated structures. (b) SAXS data, plotted as $I \cdot q^{2}$ vs. $q$, obtained for PEO-PLGA $(C x)$ solid samples. Continuous arrows pointing downward indicate peaks related to blockcopolymer length scale, while dotted arrows indicate peaks related to the PLGA(CX) complex. (c) WAXS data for PEO-PLGA and PEO-PLGA $(C X)$ solids.

two distinct levels - owing first to microphase separation between the copolymer blocks (low q, solid arrows pointing downward), and secondly to organization within the peptide-amphiphile complex domains (dotted upwardpointing arrows at higher q). The complexes with (C8) and (C12) show Bragg peaks associated with the block copolymer scale at $q^{*}=0.27 \mathrm{~nm}^{-1}$ and $q^{*}=0.28 \mathrm{~nm}^{-1}$, respectively. Higher order reflections at $q \approx 2 q^{*}$ indicate well-ordered lamellar structures with repeat spacings of $23 \mathrm{~nm}$ [PEO-PLGA(C8)] and $22 \mathrm{~nm}$ [PEO-PLGA(C12)]. Scattering from the PEO-PLGA(C18) complex shows a Bragg peak at $q^{*}=0.23 \mathrm{~nm}^{-1}$, and a very faint hint of a peak at $2 q^{*}$. An assignment of lamellar structure with a period of $27 \mathrm{~nm}$ is therefore suggested.

Correlation peaks related to the peptide/amphiphile complex blocks [indicated in Figure $3(b)$ with dashed arrows] occur at $q=2.84 \mathrm{~nm}^{-1}$ (C8), $q=1.92 \mathrm{~nm}^{-1}$ (C12) and $q=1.65 \mathrm{~nm}^{-1}$ (C18), corresponding to distances of 22 , 33 and $38 \AA$, respectively. In the case of the $\mathrm{C} 18$ complex, a well-pronounced second order peak at $q=3.38 \mathrm{~nm}^{-1}$ $\left(\sim 2 q^{*}\right)$ indicates a well-ordered lamellar arrangement. For the $\mathrm{C} 8$ and $\mathrm{C} 12$ complexes, no higher order reflections are observed, precluding the unambiguous assignment of a specific morphology for these structures.

Scattering at wide angles [Figure 3(c)] indicates a significant degree of crystallinity in all samples (with and without amphiphile complexation), due to the PEO blocks. No discernable difference exists between the scattering patterns of the various complexes, indicating little to no crystallinity in the amphiphile alkyl tails. A detailed analysis by FT-IR of C-H stretching vibrations in the alkyl tails, which otherwise would shed light on the question of amorphous vs. "solid-like" environment for the amphiphile alkyl tails, is precluded due to overlap with signal from $\mathrm{C}-\mathrm{H}$ stretching bands from PEO.

Experiments on 1:1 complexes between homopolymer PLGA and alkyl(trimethyl)ammonium surfactants (C12, C16 and C18) were reported by Ponomarenko et. al. ${ }^{[1,32]}$ WAXS results in these cases showed crystallinity in the surfactant "side chains" only for the h-PLGA(C18) case. However, FT-IR analysis suggested highly extended "solidlike" conformations for the C16 surfactants, and slightly less so for the C12 case. SAXS results and geometrical arguments showed that these complexes self-organized into lamellae consisting of alternating layers of surfactant and $\alpha$-helical peptide, with the alkyl chains fully extended, interdigitated and oriented perpendicular to the lamellar surfaces. The reported repeat distances correspond extremely well (within $1 \AA$ ) with those found in the PEO-PLGA(C12) and PEO-PLGA(C18) complexes presently investigated, indicating the adoption of the same structure. The repeat spacing found in the peptide(alkylamine) domain of the PEO-PLGA(C8), however, is less than that would be expected by linear extrapolation of the $\mathrm{C} 12, \mathrm{C} 16$ and $\mathrm{C} 18$ cases, indicating that for the $\mathrm{C} 8$ case there is some relaxation of the perpendicular, fully extended, interdigitated conformations adopted in the complexes with longer tail length. Also similar to the results presented here, the h-PLGA(C18) complex was the only one to show a well-pronounced second order Bragg peak. ${ }^{[32]}$

In light of the behavior of the PLGA(Cx) domains, it is interesting to re-visit the dependence of the block copolymer lamellar period on amphiphile alkyl tail length, the variation of which from C8 to C18 effected a modest increase of $5 \mathrm{~nm}$ in long period. Given that the length of the PLGA helices is fixed by the peptide block 
length $\left(\approx 3.45 \mathrm{~nm}\right.$ for $\left.N_{\text {PLGA }}=23\right){ }^{[28]}$ an increase in PLGA $(C \chi)$ spacing (such as occurs upon increasing amphiphile length) should not significantly alter the width of the PLGA $(C \chi)$ domains, but should rather decrease the grafting density of PEO chains at the PEO/PLGA(Cx) interface. This should effect a decrease in block copolymer long period ${ }^{[19]}$ if incompressibility is maintained in the PEO domains whereas the opposite is observed. However, subtle changes in morphology with varying amphiphile lengths may be occurring instead. For example, we have no information on the possibility that the PLGA $\alpha$-helices may be tilted with respect to the PEO/PLGA $(C x)$ interface normal direction. If this is the case, this angle may change in order to keep the PEO grafting density more or less constant. Adding to the structural complexity is the presence of semicrystallinity in the PEO block domains. For these reasons, a detailed structural model for the structures formed is not yet forthcoming. However, experiments to tease out such details are under way.

\section{Conclusion}

Hierarchical self-assembly is observed in "hairy rod"-coil peptide-synthetic hybrid block copolymer + amphiphile supramolecular complexes produced via proton transfer and subsequent ionic bonding. Ordering is observed on the scale of a few angstroms (PEO crystallinity), a few tens of angstroms [in the polypeptide(amphiphile) complex domains] and a few tens of nanometers (at the block copolymer level). In addition to demonstrating hierarchical self-assembly in supramolecularly complexed materials incorporating polypeptide structural motifs, these materials represent new systems to further the study of fundamental rod-coil block copolymer behavior. For example, the presence of amphiphiles complexed with rods is anticipated to affect both rod-rod supramolecular interactions (such as hydrogen bonding) as well as "softening" rod-rod liquid crystalline interactions (MaierSaupe). The understanding of the interplay of these mechanisms requires a systematic study in which the size of rod, coil and surfactants, as well as temperature, are suitably varied and will be addressed in a following paper.

Acknowledgements: This work was funded by the Swiss National Science Foundation. We gratefully acknowledge Prof. J.-N. Aebischer at the Fribourg College of Engineering for access to FT-IR and DSC instruments, Prof. A. D. Schlüter and Martin Colussi (ETHZ) for access to preparative GPC and Dr. S. Viale (EPFL) for useful discussions.
Keywords: biopolymers; block copolymers; self-assembly; supramolecular complexes

[1] M. Muthukumar, C. K. Ober, E. L. Thomas, Science 1997, 277, 1225.

[2] J. M. Lehn, Angew. Chem., Int. Ed. Engl. 1988, 27, 89.

[3] O. Ikkala, G. ten Brinke, Chem. Commun. 2004, 19, 2131.

[4] I. W. Hamley, "The Physics of Block Copolymers", Oxford University Press, Oxford 1998.

[5] M. Antonietti, J. Conrad, A. Thünemann, Macromolecules 1994, 27, 6007.

[6] C. F. J. Faul, M. Antonietti, Adv. Mater. 2003, 15, 673.

[7] N. Canilho, E. Kasemi, R. Mezzenga, A. D. Schlüter, J. Am. Chem. Soc. 2006, 128, 13998.

[8] N. Canilho, E. Kasemi, A. D. Schluter, J. Ruokolainen, R. Mezzenga, Macromolecules 2007, 40, 7609.

[9] N. Canilho, M. Scholl, H.-A. Klok, R. Mezzenga, Macromolecules 2007, 40, 8374.

[10] A. F. Thunemann, S. General, Macromolecules 2001, 34, 6978.

[11] T. Kato, J. M. J. Frechet, Macromolecules 1989, 22, 3818.

[12] G. ten Brinke, J. Ruokolainen, O. Ikkala, Adv. Polym. Sci. 2007, 207, 113.

[13] D. G. Kurth, P. Lehmann, M. Schutte, Proc. Natl. Acad. Sci. 2000, 97, 5704.

[14] J. Ruokolainen, J. Tanner, G. ten Brinke, O. Ikkala, M. Torkkeli, R. Serimaa, Macromolecules 1995, $28,7779$.

[15] J. Ruokolainen, R. Mäkinen, M. Torkkeli, T. Mäkelä, R. Serimaa, G. ten Brinke, O. Ikkala, Science 1998, 280, 557.

[16] M. Lee, B. K. Cho, W. C. Zin, Chem. Rev. 2001, 101, 3869.

[17] M. Reenders, G. ten Brinke, Macromolecules 2002, 35, 3266.

[18] N. Sary, L. Rubatat, C. Brochon, G. Hadziioannou, J. Ruokolainen, R. Mezzenga, Macromolecules 2007, 40, 6990.

[19] N. Sary, R. Mezzenga, C. Brochon, G. Hadziioannou, J. Ruokolainen, Macromolecules 2007, 40, 3277.

[20] H. A. Klok, S. Lecommandoux, Adv. Polym. Sci. 2006, $202,75$.

[21] H. A. Klok, J. Polym. Sci., Part A: Polym. Chem. 2005, 43, 1.

[22] S. Hanski, N. Houbenov, J. Ruokolainen, D. Chondronicola, H. Iatrou, N. Hadjichristidis, O. Ikkala, Biomacromolecules 2006, 7, 3379.

[23] A. F. Thunemann, D. Schutt, R. Sachse, H. Schlaad, H. Mohwald, Langmuir 2006, 22, 2323.

[24] A. F. Thunemann, J. Beyermann, H. Kukula, Macromolecules 2000, 33, 5906.

[25] G. Floudas, P. Papadopoulos, H.-A. Klok, G. W. M. Vandermeulen, J. Rodriguez-Hernandez, Macromolecules 2003, 36, 3673.

[26] D. S. Poche, M. J. Moore, J. L. Bowles, Synth. Commun. 1999, 29, 843.

[27] Y. Z. Yang, S. Antoun, R. M. Ottenbrite, S. Milstein, J. Bioact. Compat. Polym. 1996, 11, 219.

[28] H. Block, "Poly( $\gamma$-benzyl-L-glutamate) and Other Glutamic Acid Containing Polymers", Gordon and Breach, New York 1983.

[29] D. M. DeLongchamp, P. T. Hammond, Langmuir 2004, 20 , 5403.

[30] F. Cser, J. Appl. Polym. Sci. 2001, 80, 358.

[31] E. A. Ponomarenko, A. J. Waddon, K. N. Bakeev, D. A. Tirrell, W. J. MacKnight, Macromolecules 1996, 29, 4340.

[32] E. A. Ponomarenko, A. J. Waddon, D. A. Tirrell, W. J. MacKnight, Langmuir 1996, 12, 2169. 